\title{
Condicionantes de Género, Empleo y Trabajo y su Posible Vínculo con Afecciones y Dolencias Musculoesqueléticas y Psicosociales de Temporeras Frutícolas de Packing Agroindustrial de la Región del Libertador General Bernardo O'Higgins, Chile
}

\author{
DETERMINANTS OF GENDER, EMPLOYMENT AND WORKING AND THEIR POSSIBLE LINK WITH AND MUSCULO- \\ SKELETAL AND PSYCHOSOCIAL COMPLAINTS AND DISEASES OF FRUIT TEMPORARY WORKERS OF THE AGRO- \\ INDUSTRIAL IN LIBERTADOR GENERAL BERNARDO O'HIGGINS REGION, CHILE
}

Angélica María Valero Pinto' ${ }^{1}$ Jorge Vergara Quezada ${ }^{2}$, Cristian M. Rojas Cortés ${ }^{3}$, Lina María Quiceno Hurtado ${ }^{4}$

1. Facultad de Ciencias Politicas y Sociologia, Universidad Complutense de Madrid, España.

2. Departamento de Ingeniería Eléctrica, Universidad de Chile, Santiago, Chile.

3. Investigador adjunto de la Universidad Diego Portales, Santiago, Chile.

4. Ergónoma, Asociación Chilena de Seguridad (ACHS).

\section{RESUMEN}

El trabajo de temporera de packing representa una pieza fundamental en la creciente agro-exportación chilena; sin embargo, el daño físico y psicosocial que sufren estas trabajadoras es observado en el largo plazo, lo cual hace que las actuales medidas que buscan protegerlas resulten insuficientes. El objetivo de esta investigación es detectar y relacionar posibles causas de dolencias musculoesqueléticas y psicosociales de las temporeras a partir de una perspectiva global que integra: género, condicionantes de trabajo y empleo. La metodología para detectar y medir factores de riesgo asociados al trabajo temporero se diseñó a partir de la realización de entrevistas en profundidad y encuestas a 132 temporeras de empresas agroindustriales con más de 200 trabajadores de la VI región. Los resultados obtenidos muestran que las exigencias en trabajo de packing, más las exigencias propias por condición de mujer, son aspectos que deterioran la salud física y mental de las trabajadoras. Las conclusiones y discusiones derivaron en la necesidad de buscar mecanismos que hagan conciliar salud y calidad de vida de temporeras frente a la necesidad de productividad de la empresa, dado que las actuales condiciones de trabajo y empleo se levantan mayormente a partir del fomento productivo empresarial.

(Valero A, Vergara J, Rojas C, Quiceno L, 2015. Condicionantes de Género, Empleo y Trabajo y su Posible Vínculo con Afecciones y Dolencias Musculoesqueléticas y Psicosociales de Temporeras Frutícolas de Packing Agroindustrial de la Región del Libertador General Bernardo O’Higgins, Chile. Cienc Trab. May-Ago; 17 [53]: 107-114).

Palabras claves: GÉNERO, FACTORES DE RIESGO, DOLENCIA MUSCULOESQUELÉTICA, ASPECTOS PSICOSOCIALES, ESTRÉS PSICOLÓGICO.

\section{ABSTRACT}

Female temporary packing workers play a key role in the growing agro-export business in Chile. Yet, the physical and psychosocial damage suffered by those workers can be observed in the long term. This very fact proves the current measurements that seek to protect them to be insufficient. This research aims at identifying possible causes of musculoskeletal and psychosocial diseases of the temporary workers from a global perspective that seeks to integrate issues of gender, as well as of labour and employment conditions, putting them in connection to those causes. The methodology for detecting and measuring those risk factors associated to temporary job was designed after conducting interviews and surveys to one hundred and thirty two (132) temporary agribusiness companies with more than two hundred (200) workers in the province of Libertador General Bernardo 0'Higgins. The obtained results suggest that the specific requirements of the packing job, in addition to the specific requirements of the gender, have an active role in the spoil of both physical and mental health of female temporary workers. The conclusions drawn from these results point to the need for finding mechanisms to reconcile health and well-being criteria related to the workers, and productivity criteria related to the business; since the current conditions of employment are mostly raised in view to the corporate productive model of development.

Keywords: GENDER, RISK FACTOR, MUSCULOSKELETAL PAIN, PSYCHOSOCIAL ASPECTS, STRESS PSYCHOLOGICAL.
Correspondencia / Correspondence:

Angélica Valero Pinto

Santo Domingo 498, depto. 904,

Santiago, Chile.

e-mail: angevale@gmail.com

Tel.: +56988230899

Recibido: 12 de Abril 2015 / Aceptado: 25 de Junio 2015

\section{INTRODUCCION}

Las favorables condiciones climáticas en Chile hacen de la agroexportación uno de los pilares del desarrollo económico del país. ${ }^{1,2}$ Dentro de esta cadena, la trabajadora temporera de packing resulta un eslabón fundamental, siendo requerida por la productividad empresarial debido a sus características relacionadas, intrínsecamente, con lo femenino y adquiridas en el trabajo doméstico. Tales 
características demandadas en el packing tienen relación con la meticulosidad, rapidez, mayor flexibilidad a los cambios de funciones, cuidado con la fruta, entre otras. ${ }^{3-6}$

Las principales particularidades del trabajo de packing son los requerimientos de movimientos rápidos y sobreexigentes, así como de capacidad para trabajar en ambientes de gran presión psicológica. El problema de este trabajo es que el daño físico (musculoesquelético) y mental (psicosocial) es acumulativo y se manifiesta en el tiempo, teniendo como consecuencia subdiagnóstico y subregistro de enfermedades profesionales, así como la invisibilización de los factores de su origen. ${ }^{7-9}$

La escasa investigación existente, específicamente sobre aparición de sintomatología musculoesquelética y psicosocial en packing, se caracteriza por homogeneizar los factores de riesgo de las temporeras de packing con otros rubros agroindustriales, simplificando las causas de este problema al lugar y/o las condiciones de trabajo. Estos estudios, en general, buscan principalmente detectar sintomatología en los miembros superiores -manos, brazos y hombros ${ }^{10-13}$-y visibilizar la sobre-precarización de condiciones laboral. ${ }^{14,15}$ En el caso de Chile existe un conjunto de marcos regulatorios para amortiguar la relación salud-trabajo, tales como: Código del Trabajo ${ }^{16}$; Ley $\mathrm{N}^{\circ}$ $16.744^{17}$; Decreto Supremo 109 ${ }^{18}$; Decreto 594 ${ }^{19}$; Ley $\mathrm{N}^{\circ} 20.123^{20}$; Ley $\mathrm{N}^{\circ} 19.404^{21}$; y la Ley $\mathrm{N}^{\circ} 20.005 . .^{22}$ A pesar de lo anterior, no se cuenta con programas de prevención y estudios epidemiológicos sistemáticos y específicos de packing que permitan identificar causas e impactos reales del trabajo de packing en la salud física y mental de las temporeras. ${ }^{7,9,23}$

El análisis actual de esta problemática deja a la temporera solo como una pieza dentro del engranaje de la empresa, sin considerar su identidad, diversidad y carga histórica (vigente) de mujer. Considerando esta falencia, es que la presente investigación tiene como objetivo indagar, desde una perspectiva de género, posibles vínculos existentes entre condicionantes de género, condiciones de empleo y condiciones de trabajo, para detectar causas en la aparición de dolencias y afecciones en salud musculoesquelética y salud psicosocial de trabajadoras temporeras de packing de la agroindustria frutícola de la Región del Libertador General Bernardo O'Higgins, Chile.

Para entender la importancia del enfoque de género para esta investigación, es necesario señalar que la feminización del mercado de trabajo agrícola se ha dado gracias a la aplicación de un modelo de flexibilidad laboral ${ }^{24,25}$, realizado mediante la aplicación de mecanismo de contratación y formas de pago flexibles legalmente establecidos, según las normas laborales vigentes. No obstante, si bien esta flexibilización ha permitido el ingreso de mujeres al trabajo productivo, también ha significado una sobre-precarización de sus condiciones laborales. ${ }^{15,26}$ Tal flexibilización impacta negativamente en la salud física y mental de la mujer temporera, ya que no considera necesidades propias de ésta, tales como: organización del trabajo, diseño de los puestos de trabajo ${ }^{27}$, protección de salud en periodos de inactividad (cesantía, vejez, enfermedad propia o de algún miembro de su grupo familiar). Al mismo tiempo, el proceso de asalarización femenina se enmarca en un contexto de persistencia de la división sexual y doméstica del trabajo tradicional, lo cual introduce una tensión derivada de la necesidad de compatibilizar el empleo asalariado con las variadas responsabilidades familiares. Esta sobrerresponsibilización se engloba en el concepto de doble presencia. ${ }^{28}$ Además, los datos entregados por el primer Organismo Técnico Intermedio de Capacitación orientado al sector silvoagropecuario (OTIC AGROCAP) ${ }^{25}$ indica que las mujeres tienen empleos que, en promedio, son de menor duración que los de los hombres.
Son pocos los estudios que abordan los vínculos entre: salud mental, salud musculoesquelética, condiciones de trabajo, trabajo remunerado y no remunerado, y prácticas de prevención, desde un análisis con perspectiva de género. ${ }^{14,28-33}$ Sin embargo, estos estudios han permitido validar el perjudicial efecto de la flexibilización-precarización del trabajo en situaciones que a diario viven las mujeres de la agroindustria. Es así como Willson y Caro $^{14}$ muestran los desafíos que enfrenta la mujer al intentar conciliar su vida laboral y familiar sobre un espacio altamente complejo como lo es el rural. Por otro lado, el vínculo entre calidad del trabajo, empleo y salud mental ${ }^{34}$ muestra que las nuevas y exigentes formas de organización de la producción y modalidades de relaciones laborales son determinantes en la morbilidad de la población adulta, siendo la mujer la más afectada debido, principalmente, a doble presencia, economía de los cuidados y división sexual del trabajo. Garay ${ }^{35}$ muestra que, en el caso de las mujeres temporeras rurales, el riesgo de padecer afecciones en la salud mental es aún mayor ${ }^{35,36}$ en relación al resto de mujeres. Además, la información arrojada por La Primera Encuesta Nacional de Empleo, Trabajo, Salud y Calidad de Vida de los Trabajadores y Trabajadoras en Chile (ENETS) 2009-2010³, concluyó que la inequidad es un fenómeno complejo y muchas veces invisible a la sociedad, afectando a los grupos más vulnerables.

La importancia de estos estudios es que no solo visibilizan las inequidades por razón de sexo a las que se deben enfrentar las mujeres rurales, sino que además muestran la necesidad de un nuevo enfoque para analizar las dolencias físicas y mentales de los/las trabajadores/ as de la agroindustria. Siguiendo esa línea, es que esta investigación busca desarrollar mecanismos que hagan conciliar salud y calidad de vida de temporeras de packing frente a la necesidad de productividad de la empresa; dado que las actuales condiciones de trabajo y empleo se levantan mayormente a partir del fomento productivo empresarial.

\section{METODOLOGÍA}

\section{Diseño de investigación}

Se utilizó una metodología mixta basada en un enfoque cuantitativo (encuesta) y un enfoque cualitativo (entrevista). El enfoque cuantitativo tuvo como finalidad abordar y cuantificar aspectos tales como: condición física general; condición hombro, brazo, mano; ambiente de trabajo; y seguridad laboral, siendo todos estos aspectos vistos desde la perspectiva de género. El enfoque cualitativo tuvo como finalidad validar y complementar los resultados obtenidos en la encuesta, permitiendo profundizar en aspectos de género comúnmente invisibilizados en las trabajadoras de packing y difícilmente cuantificables.

\section{Participantes}

Se define como muestra un número a determinar de trabajadoras de packing agroindustrial frutícola de una o más empresas de la Región del Libertador General Bernardo O'Higgins que conste en las bases de datos de la Asociación Chilena de Seguridad (ACHS) que hayan presentado sintomatología musculoesquelética y/o psicosocial en un período reciente -año 2012 o 2013-, considerando las posibilidades de acceso a la empresa y cumpliendo los requerimientos metodológicos que hagan posible validar el análisis posterior.

En general, los packings dependen de empresas medianas o grandes, es decir, con mayor capital. Dada la mayor visibilidad y concentración de trabajadores/as en un solo lugar físico (packing), susceptible a una mayor fiscalización, se puede colegir que en estos espacios 
laborales existiría un mayor nivel de formalización de la relación contractual. En base a esto último, los criterios de selección de las mujeres temporeras a encuestar fueron: (i) Mujeres temporeras de la VI Región, (ii) Trabajadoras de packing pertenecientes a empresas de más de 200 empleados. La estimación de la muestra de mujeres temporeras necesarias para la realización de la encuesta fue obtenida utilizando un error muestral de un $2 \%$, un intervalo de confianza del 95\%, así como una proporción de un 99\% de individuos que poseen, en la población, la característica buscada en este estudio $(p=0,99)$. La asignación de este último valor se justifica ya que las encuestas se realizaron solo a empresas que cumplían los requisitos del estudio. El tamaño de la población se obtuvo a partir de los datos entregados por la encuesta CASEN 2009..$^{38-40}$ El número mínimo de encuestas necesarias calculado fue 93, sin embargo, se prefirió aumentar el número a 132 encuestas realizadas a 3 empresas agroindustriales. En el enfoque cualitativo, se realizaron 49 entrevistas que se dividen en cuatro grupos: (i) 25 entrevistas a informantes claves ${ }^{41,42}$, que corresponden a personas pertenecientes a: ONG, centros de investigación, organizaciones de empresariado agrícola, entre otros. (ii) 8 entrevistas a informantes porteros ${ }^{41,42}$, los cuales corresponden a personas pertenecientes a asociaciones gremiales, sindicatos, entre otros. (iii) 8 entrevistas a temporeras que se encontraban retiradas de las labores de packing, y (iv) 8 entrevistas a temporeras que se encontraban realizando actualmente labores de packing. El número de entrevistas fue determinado por el principio de saturación y las personas entrevistadas fueron seleccionadas para ser válidos representantes en cada uno de los grupos mencionados anteriormente. La información buscada en cada uno de los grupos fue:

Grupo (i) y (ii): contacto con empresas y temporeras, obtención de información relevante para el diseño de la encuesta y visión que las empresas o sus representantes tienen sobre el trabajo de packing. Grupo (iii) y (iv): obtención de información desde la propia temporera en relación a su salud (física y mental) desde dentro y fuera de la empresa.

\section{Encuesta}

La encuesta fue diseñada para abarcar las cinco dimensiones de investigación que son: (i) género, (ii) factores musculoesqueléticos, (iii) factores psicosociales, (iv) condiciones de empleo, y (v) condiciones de trabajo. Para ello se utilizaron tres cuestionarios existentes en la literatura y que han sido validados nacional ${ }^{37-43-45}$ e internacionalmente ${ }^{46-48}$ de la siguiente forma: (i) SF12 en su versión española $2.0^{46,47}$ utilizado para medir la percepción de la salud de la temporera, cuantificando en dos medidas el grado de salud física (Índice de Salud Física (ISF)) y el grado de salud mental (Índice de Salud Mental (ISM)) de ésta. El algoritmo utilizado para obtener estas medidas fue obtenido de Social Security Administration. ${ }^{49}$ El valor de los índices finales se representan en porcentajes, donde un 50\% (con una variación de $\pm 10 \%$ ) representa un nivel de salud normal. (ii) Quick-Dash ${ }^{48}$ utilizado para medir la condición hombro, brazo y mano, y estado físico general de la temporera. Además, se utilizó el módulo de trabajo del Quick-Dash, que mide la discapacidad que afecta a la temporera en el trabajo. Los indices del Quick-Dash y Quick-Dash módulo trabajo, se entrega en porcentaje, siendo un 0\% el indicador de ausencia de dolores en el tronco superior del cuerpo de las temporeras, y un 100\% indicador de imposibilidad de movimiento producto de dolores en el tronco superior. (iii) ENETS 2009-2010 ${ }^{37}$, de la cual sólo se utilizaron algunas preguntas que estaban relacionadas con condiciones de trabajo y forma de remuneración. Además de los tres cuestionarios mencionados, se agregaron preguntas especificas relacionadas con aspectos de género y prevención de accidentes en las empresas. Considerando que la encuesta fue realizada a mujeres temporeras en su lugar de trabajo, en períodos de alta demanda laboral, con poco tiempo para responder la encuesta, fue necesario reducir la cantidad de preguntas hasta un número mínimo de 79 preguntas. Al finalizar la toma de encuesta, se detallaron aspectos relevantes relacionados con las trabajadoras. Los aspectos relevados fueron: (i) ¿Tuvo que asistir a la temporera para responder la encuesta?, en donde se detectó la presencia de grados de analfabetismo (absoluto o funcional) en la temporera, y (ii) Comentarios o cuestiones importantes a considerar que hayan llamado su atención y que considere necesarios de relevar en relación a esta jornada de toma de muestra. La toma de la encuesta duró alrededor de 30 minutos.

\section{Entrevista}

Este enfoque permitió conocer la propia visión de la temporera en relación a su actual estado de salud y su percepción acerca de las posibles causas de sus dolencias. Las entrevistas fueron realizadas en lugares acordados con cada uno/a de los entrevistados/as y los temas abordados fueron: (i) doble presencia, (ii) estrés laboral versus estrés doméstico, (iii) posibles causas de dolencias musculoesqueléticas y psicosociales del trabajo de packing, condiciones de empleo y trabajo. Las entrevistas adoptaron la forma de conversación hilada entre el/la investigador/a y la temporera, sobre la manera que esta tiene de comprender, experimentar y vivenciar el mundo y la realidad cotidiana, considerando aquellos aspectos y categorías de las cinco dimensiones de investigación. Por otro lado, la información de las temporeras fue cotejada con la información de los entrevistados representantes de las empresas.

\section{Análisis de resultados}

Se obtuvieron las medidas de los índices de los cuestionarios SF12v2, Quick-Dash y Quick-Dash módulo trabajo, siendo estos cruzados con el factor edad de cada temporera con el fin de medir efectos en salud a través del tiempo. Se realizó una estadística descriptiva univariada usando distribución de frecuencias, calculando estadísticos de resumen de media y porcentajes. Esta información numérica es complementada con información entregada por las temporeras en cada una de las entrevistas. El análisis de los resultados se realizó para cada una de las cinco dimensiones de investigación.

\section{RESULTADOS Y ANÁLISIS}

\section{Caracterización de las trabajadoras encuestadas}

Las edades de las mujeres fluctúan entre 18-73 años, concentrándose las dos terceras partes de este grupo entre los 37-64 años. Las actividades desempeñadas en packing por las mujeres encuestadas son: (i) Selección (69,7\%), (ii) Embalaje (40,9\%), (iii) Limpieza (35,6\%), (iv) Pesaje (12,9\%). El 28,8\% de las temporeras indicó ser la única sostenedora del grupo familiar, mientras que un 50,7\% indicó recibir ayuda económica de su pareja, hijo o hija o algún familiar cercano. La cantidad promedio de integrantes del grupo familiar de cada temporera es de aproximadamente 3,5 a 4 personas. El 2,27\% de las temporeras indicó que durante el último año solo ha trabajado en su actual empresa, el 71,96\% indicó que ha trabajado en otra empresa, y el 16,7\% indicó que ha trabajado en 3 o más empresas. El 65,2\% de las temporeras trabaja más de 8 meses durante el año (no limitado solo al ámbito agroindustrial). 
Un 72,7\% de temporeras indicó trabajar de día con jornada laboral completa. Finalmente, dentro de los trabajos agrícolas que las temporeras han realizado durante el último año: un 12,9\% de temporeras trabajó en cosecha, 11,4\% trabajó en limpieza, un 94,7\% trabajó en packing y un $6,1 \%$ de temporeras trabajó en otras labores tales como desbrotar e injerto de parras.

\section{CONDICIONANTES DE GÉNERO}

\section{Economía de los cuidados}

Según las temporeras, las normas sociales relativas a la división sexual del trabajo reproductivo (doméstico y de cuidado) no se han modificado, siendo un ámbito de responsabilidad exclusiva de las mujeres. El 78,03\% de trabajadoras encuestadas indicaron ser jefas de hogar. A la jornada laboral de las temporeras se superpone, muchas veces, la preparación de comidas en las madrugadas, y una labor de ordenamiento de la casa y lavado de la ropa en días libres.

\section{Conciliación vida laboral - vida familiar - vida social}

El 82,35\% de mujeres indicó que nunca o muy rara vez cuenta con tiempo libre o de ocio. El 93,2\% de las mujeres trabajadoras consideraba que su tiempo libre corresponde al tiempo que dedica a las labores del hogar o a agasajar a su familia con comidas o atenciones. Las tareas relativas al cuidado infantil se destacan como una preocupación constante $\mathrm{y}$, muchas veces, no del todo bien resueltas. Las trabajadoras indicaron que para sobrellevar las tareas del cuidado familiar versus responsabilidades laborales y familiares necesitan recurrir a redes de parentesco, vecinos, tutela de la hija mayor y/o el uso de sistemas informales de cuidado pagado, que conllevan costos económicos y emocionales que recaen en las propias temporeras. A pesar de esto, solo el 34,1\% de las temporeras indicó que cuenta con el apoyo de alguien que ayuda en las labores del hogar.

\section{Doble presencia}

El 86,36\% de temporeras indicó que mientras trabaja está pensando en exigencias domésticas y familiares, a pesar de tener a alguien que las reemplace en el trabajo reproductivo. Según informan las temporeras, sus hijos/as, al quedar al cuidado de familiares, vecinos, amigos, o instituciones del Estado presentan mayores riesgos de vulnerabilidad tales como: consumo de drogas, embarazos adolescentes, accidentes, violencia sexual, entre otras. Todas las mujeres que tienen hijos/as, indicaron que viven con mucha culpa sus ausencias.

\section{Auto-postergación}

Un 32,58\% de mujeres temporeras indicó contar con apoyo (doméstico) y compresión de su pareja en periodo de alta demanda laboral. Las temporeras concuerdan en señalar la permanente postergación que hacen del mejoramiento de su salud; "Siempre habrá otro que atender primero: padres, hijos u otros familiares”. El 78,79\% de temporeras indicó que siempre o a veces se auto-medica para disminuir los dolores físicos, mientas que el 30,30\% indica auto-medicación para sentirse más animada.

\section{EXIGENCIAS FISICAS DEL TRABAJO: FACTORES MUSCULOESQUELÉTICOS}

De las 132 mujeres trabajadoras temporeras encuestadas, solo 116 respondieron la totalidad de las preguntas del SF12. De estas, el 75\%
(87/116) obtuvo un ISF entre 40 y 59,9, indicando una salud física normal de acuerdo a la población de referencia. El 25\% restante (29/116) mostró un valor de ISF menor a 40, que indica salud física deteriorada. De estas últimas, un 44,8\% (13/29) realiza más de dos actividades en la empresa entre las que se encuentra: limpieza (13/29), pesaje (3/29) y embalaje (13/29). Un análisis del ISF por edad es presentado en la Figura 1, donde se observa que no existe una correlación directa entre edad y estado de salud físico de la temporera (valor de ISF). Según indican las temporeras, muchas de las mujeres de mayor edad son "ayudadas" por sus compañeras más jóvenes en el desarrollo de labores dentro de la empresa.

Para resultados de Quick-Dash, se debe tener en cuenta que sólo 127 trabajadoras (de las 132) contestó el mínimo de preguntas del cuestionario. De estas, 81,89\% (104/127) obtuvo un valor de Quick-Dash mayor a 45 indicando que el estado físico del tronco superior de su cuerpo tiene dolencias que impide realizar normalmente actividades dentro y fuera de su lugar de trabajo. Para indagar respecto a la causa laboral detrás de la dolencia, se analiza el índice del Quick-Dash módulo trabajo; 130 de las 132 temporeras encuestadas respondieron las 4 preguntas del módulo, observándose igual tendencia de mujeres con fuertes dolencias en el tronco superior del cuerpo. Existe un 28,46\% de mujeres (37/130) con índice de Quick-Dash módulo de trabajo con índice superior a 95 .

En Tabla 1 se observa que las temporeras (de mayor edad) presentan bajos valores de índice de Quick-Dash y Quick-Dash módulo trabajo. Es importante destacar que el Quick Dash no realiza ninguna indagación específica sobre las causas detrás de las dolencias físicas del tronco superior, es decir, no detecta el efecto acumulativo de las dolencias físicas provocadas por las labores de packing, sino más bien son las propias trabajadoras encuestadas las que indican que todo el daño físico que experimentan en el tronco superior del cuerpo (y que se ve reflejado en las respuestas de Quick Dash), se debe casi exclusivamente a los trabajos de packing que han desarrollado la mayor parte de su vida laboral para múltiples empresas agroindustriales.

\section{Figura 1.}

Gráfico de dispersión que relaciona el índice de salud física y la edad de 116 temporeras encuestadas en las tres empresas estudiadas.

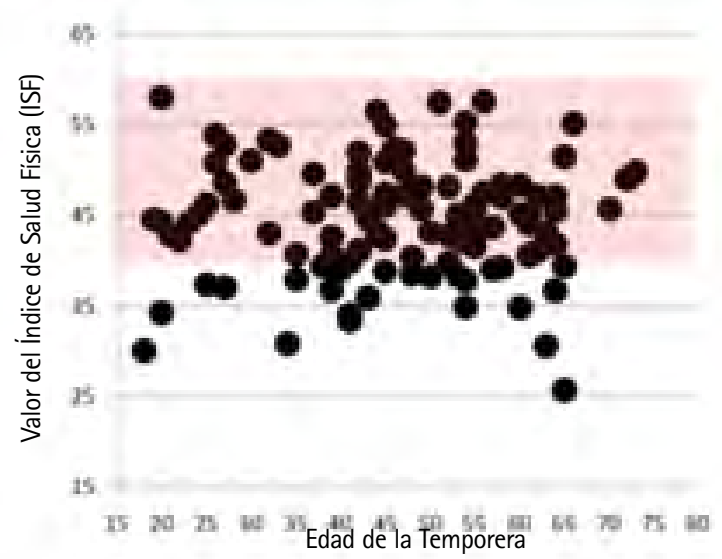

Cada punto indica la edad y valor del índice de salud de la temporera. La zona de color rojo indica el intervalo del índice de salud física donde la media de la población de referencia $(50 \pm 10)$ posee una salud física normal. 
Tabla 1. Matriz de frecuencia de los índices de quick-dash (qd) y quick-dash módulo trabajo (qdt) versus la edad de las temporeras.

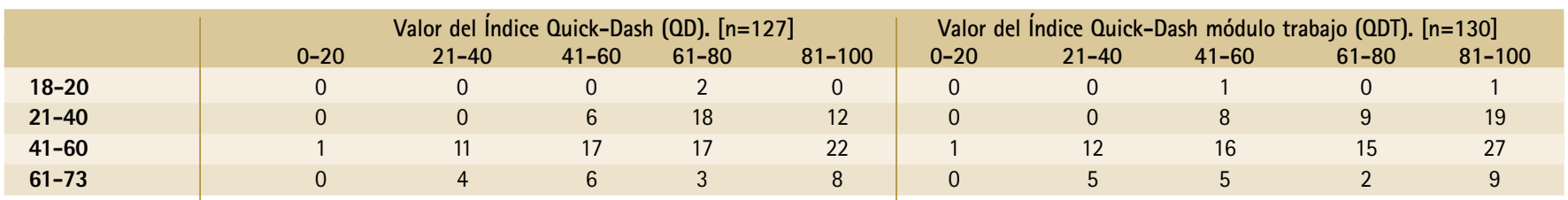

Cada celda indica la cantidad de temporeras que se encuentra sobre cierto rango de edad y rango del índice. La cantidad total de temporeras analizadas en el qd y qdt está dado por el valor $n$.

\section{EXIGENCIAS PSICOLÓGICAS DEL TRABAJO: FACTORES PSICOSOCIALES}

\section{Demanda física como factor de riesgo psicosocial}

Según las trabajadoras, el ritmo de trabajo debe ajustarse a la cantidad de fruta que entrega la cinta transportadora. Este aspecto se encuentra estrechamente relacionado y abordado en dato sobre exigencia atencional: el 53,8\% de las temporeras indicó que su trabajo de packing la obliga a estar tan concentrada que no puede pensar en otra cosa. A esta demanda psicológica se suma el constante monitoreo de supervisores como mecanismo de presión hacia las temporeras para evitar distracción, asegurar ritmos acelerados de producción y el cumplimiento de metas.

La Figura 2 relaciona el valor del ISM con la edad de las temporeras. De este gráfico se observa que el deteriorado nivel de salud mental de las temporeras no tiene relación directa con la edad. El ISM indica que el 61,2\% (71/116) de temporeras está por debajo de zona considerada estado normal de salud mental (zona roja de La Figura 2).

\section{Relación hostil con las jefaturas}

El 45,5\% de temporeras respondió que frecuentemente se ha sentido presionada o intimidada por algún jefe. Además, indican que este tipo de hechos es permitido por un buen número ellas (trabajadoras) producto del fenómeno de la naturalización de violencia de género en sus propias cotidianeidades, considerándose, en tanto, aspectos no relevantes y propios de una relación jefe-subordinada.

Figura 2.

Gráfico de dispersión que relaciona el índice de salud mental y edad de 116 temporeras encuestadas en las tres empresas estudiadas.

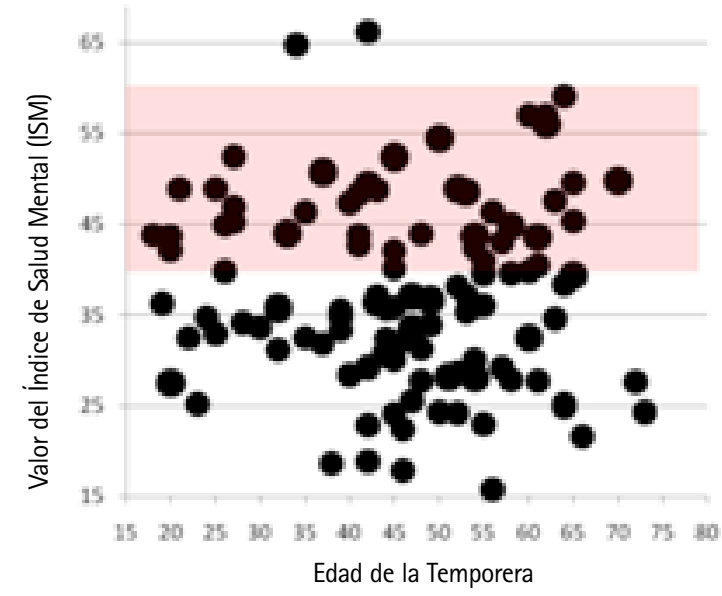

Cada punto indica la edad y valor del índice de salud de la temporera. La zona de color rojo indica el intervalo del índice de salud mental donde la media de la población de referencia $(50 \pm 10)$ posee una salud mental considerada normal.

\section{Discriminación y acoso laboral}

Según las entrevistadas, las trabajadoras jóvenes son objeto de regalías y "galanteos" constantes de parte de jefaturas masculinas; observándose hechos tales como destinación de trabajadoras jóvenes a posiciones de mayor visibilidad y acceso; y, por el contrario, invisibilización de trabajadoras de mayor edad. En el packing, el rechazo o rebeldía hacia conductas acosadoras de superiores puede implicar, según las propias temporeras, menos descarga de productos en las líneas y, con ello, menor salario. La discriminación que sufren algunas temporeras por su apariencia física, unida a la mala capacidad de adaptación y baja autoestima de estas, constituyen factores de riesgo de depresión.

\section{Falta de claridad sobre los roles}

Las temporeras indican que no existe delimitación clara de sus actividades en la empresa (desconocimiento de actividad específica que se realizará durante jornada laboral). Esto produce rabia, frustración, impotencia y estrés. El 51,5\% de trabajadoras realiza más de 2 labores diarias dentro de la empresa.

\section{Relaciones entre compañeras}

El 74,24\% de temporeras indicó que frecuentemente siente el apoyo o colaboración de sus compañeras para realizar el trabajo. Este factor se reconoce como de "protección" frente a negatividad de condiciones de trabajo. Por otro lado, se advierte que las nuevas estrategias y técnicas de pagos o bonificaciones de las empresas por trabajo individual genera una alta competitividad entre las propias compañeras minando el factor mencionado (apoyo mutuo y grupal).

\section{CONDICIONES DE EMPLEO}

\section{Contrato}

El 98,5\% de trabajadoras manifiesta que la forma de contrato es de tipo escrito. El 90\% de trabajadoras indica no haber leído el contrato cuando lo firmaron (se solicitó firma cuando se encontraban en faena). El 100\% de trabajadoras tiene contrato por obra o faena, que puede ser finalizado o renovado según necesidad de empresa. Además, la empresa maneja aspectos contractuales de: labor que debe desarrollar la trabajadora, forma de pago de remuneraciones, horarios de trabajo (horas extras y días de descanso), y tiempos de duración del contrato.

\section{Remuneración a percibir}

El $81,82 \%$ de temporeras indica que su sueldo se compone de "sueldo base más bonos por producción, menos descuentos". Las complejas y poco estandarizadas medidas de trabajo dificultan el cálculo de la remuneración final. Estas medidas se complejizan más al considerar que la mayoría de las empresas tiene sus propias unidades de medida. Otra fuente de confusión es el pago de remuneraciones en forma individual o grupal. La dificultad del pago en 
base a cálculo grupal radica en que los equipos de trabajo no son los mismos durante el transcurso del mes, resultando difícil retribuir correctamente el trabajo de cada temporera. El salario promedio entre las trabajadoras temporeras agrícolas oscila alrededor de los $\$ 180.000$ líquidos.

\section{Jornada laboral}

A pesar que las temporeras indican sobre el pago de días extras de trabajo, también plantean carecer de posibilidades para negarse a trabajar en tales horarios y días, viéndose amenazadas por una posterior no renovación de contrato.

\section{Salud y previsión social}

Todas las trabajadoras admiten que es el empleador el que les cotiza en AFP. El 93,4\% de trabajadoras dijo que en caso de problema de salud es atendida en servicio público. En caso de tener alguna dolencia dentro de horas de trabajo, el 82,5\% de las temporeras manifestó inclinarse por invisibilización de malestar a través del uso de algún medicamento, a la vez que percibir considerable presión de parte de supervisores para no enfermar ni decaer.

\section{Participación sindical}

El 100\% de trabajadoras indicó no participar en sindicatos. El temor entre las trabajadoras de ser catalogadas como "conflictivas" se comprende cuando tal calificación es sinónimo de desempleo (no recontrato). Tal mecanismo de registro sería una dinámica habitual utilizada por empresas del rubro, traduciéndose en impedimento para que mujeres "rotuladas" puedan volver a ser empleadas por la agroindustria.

\section{CONDICIONES DE TRABAJO}

\section{Comités paritarios}

Un 45,5\% indicó conocer existencia de Comité Paritario, frente a un 25\% que indicó desconocimiento. Asimismo un 48,5\% indicó desconocimiento respecto de la finalidad de éste.

\section{Percepción de riesgo de accidentes de trabajo y enfermedades profesionales}

De acuerdo a lo abordado en las entrevistas a temporeras retiradas de labores de packing, se observó que efectos en salud física y psicosocial no son detectables en periodos de actividad de la temporera (cuando se encuentra empleada). Por lo tanto, las dolencias que aparecen en periodos de "desempleo" o inactividad (por necesidad de la empresa) no se reconocen como accidentes de trabajo, ni enfermedad profesional. Constituyéndose así un caldo de cultivo -entre otros aspectos- para la automedicación, el autodiagnóstico y el subregistro de enfermedades profesionales.

\section{Analfabetismo}

Un 11,4\% de temporeras posee analfabetismo absoluto (no sabe leer ni escribir). Un 60,12\% de trabajadoras presenta analfabetismo funcional, reflejado, entre otras cosas, en que la trabajadora, no obstante saber leer no entiende las señalizaciones preventivas al interior de la empresa, ni para qué sirven, no prestando mayor importancia. Esta falta de interés, producto del analfabetismo funcional, también se refleja en la poca importancia que se le da a la existencia de comités paritarios, no entendiendo para qué sirven y desconociendo la ayuda que les puede representar.

\section{Acceso a servicios higiénicos}

A pesar de que la distancia entre baños y puestos de trabajo cumple con las normas legales, la alta demanda de trabajo de las temporeras, sumado a la constante presión de supervisores (para que se utilice la mínima cantidad de tiempo para baño), es causante de tres tipos de daño físico: (i) Infección urinaria, producto de evitar el baño en la jornada laboral. (ii) infecciones genitales y contaminación de la fruta, producto de la manipulación de fruta sin usar guantes sumado a la urgencia por regresar al puesto de trabajo tras el baño, sin lavar manos evitando pérdida de tiempo. (ii) daños físicos: por la constante vigilancia de supervisores que obliga a las temporeras ir lo más rápido posible al baño.

\section{Implementos de seguridad inadecuados}

Las trabajadoras indican que los implementos de seguridad presentan desgaste y fatiga de material dado el constante uso. Los empleadores entregan elementos de protección bajo la idea de cumplir con la normativa vigente intentando a la vez, reducir costos. Bajo dicha lógica los implementos no logran proteger lo suficiente, generando, muchas veces, problemáticas como hongos en pies, ampollas por roce, o entumecimiento de zonas del cuerpo por implementos de mayor o menor tallaje. En labores de limpieza que realizan las temporeras, se indica que rara vez se hace entrega de guantes o mascarillas de protección frente a productos limpiadores (químicos) o frente a inhalación de compuestos químicos.

\section{Elementos y medios materiales necesarios para realizar el trabajo}

Las herramientas y puestos de trabajo son diseñados utilizando parámetros anatómicos estándares sin tomar en cuenta distintas capacidades físicas, contextura muscular o edad de trabajadoras.

\section{Lugar de trabajo y entorno inmediato}

Las largas jornadas de trabajo de pie generan sobre-exigencias físicas principalmente en piernas y parte baja de espalda. A pesar de que tales dolencias escapan a los límites de esta investigación, precisamente son estas las que generan un caldo de cultivo para la adopción de malas posturas en lugar de trabajo, resultando sobre-exigencias en el tercio superior del cuerpo (hombro, brazos y manos) de la temporera. A esto se suma los periodos de demanda laboral y entornos de trabajo con exposición al frío o calor. Aproximadamente un tercio de temporeras manifestó presentar sensación de encierro producto de permanecer mucho tiempo dentro de packing.

\section{Tiempos de colación y descanso}

El 100\% de las mujeres indicó que los horarios de colación corresponden al periodo legal de 1 hora; sin embargo, en dicho tiempo no se contempla: (i) tiempo de traslado desde el puesto de trabajo al comedor, (ii) retrasos en los tiempos de detención de máquinas y mangas (lanzan fruta incluso tras haber comenzado la hora de colación), (iii) tiempo de espera en filas de comedor, donde en casos extremos no se alcanza almorzar, (iv) regreso unos minutos antes al puesto de trabajo debido a que la máquina comienza a surtir de fruta minutos antes de cumplida la hora de colación.

\section{Lugar y tiempo de descanso}

El 32,6\% del total de trabajadoras señala que el poco tiempo libre del que disponen lo destinan para estar con su familia y para intentar descansar. Esto significa nulo tiempo para ellas mismas, atender sus necesidades específicas, ocio, entretención o generación de relaciones propias de vida social. 


\section{CONCLUSIONES}

Tradicionalmente, la gran mayoría de las medidas de protección para la temporera de packing se encuentran destinadas a prevención de accidentes laborales y de enfermedades profesionales. Sin embargo, en esta investigación se pudo detectar que tales medidas resultan insuficientes. La perspectiva de género aplicada al estudio ha permitido relevar importantes aspectos que permiten visualizar posibles causas tras afecciones y dolencias musculoesqueléticas y psicosociales de trabajadoras agrícolas de temporada de la Sexta Región de Chile.

Dentro de las conclusiones más relevantes se puede mencionar:

- Analfabetismo: Grave factor de riesgo que afecta la comprensión de las instrucciones sobre seguridad entregada por la empresa. Pudiera ser un factor estrechamente relacionado a la frustración evidenciada por la temporera a la hora de tener que realizar funciones que, si bien pudieron estar estipulados en contrato, no fueron comprendidos o leídos.

- Subdiagnóstico y subregistro de enfermedades profesionales: El daño musculoesquelético y psicosocial es acumulativo y progresivo a través de los años, por lo que la necesidad de un correcto diagnóstico médico resulta de mucha relevancia. Entre las temporeras abunda la automedicación y el autocuidado para cumplir con sus responsabilidades diarias.

- Modalidad de pago: Las complejas formas de calcular el sueldo a fin de mes genera en temporeras sobre-exigencias físicas y estrés constante.

- Doble presencia y economía de los cuidados: Estrés de las trabajadoras por la vulnerabilidad de sus hijos y de otros familiares dependientes de ella.

- Ineficiencia/limitación de los actuales instrumentos (encuestas): Los actuales instrumentos de encuesta, por sí mismos, no pueden detectar las posibles causas detrás de todas las dolencias o afecciones de las temporeras, resultando con ello imposible conocer los efectos acumulativos de los trabajos agroindustriales.

- Prevalencia de dolores musculoesqueléticos: A pesar de que las actuales medidas de seguridad laboral intentan prevenir dolencias y afecciones en el packing, existen importantes aspectos que difi- cultan la detección temprana de dolencia y afecciones musculoesqueléticas en las temporeras.

- Desplazamiento efecto/causa: Los índices entregados por los cuestionarios representan un estado actual de salud de la temporera, por lo que resulta casi imposible detectar las verdaderas causas de las dolencias de la temporera.

- Errada concepción de dolencia muscular: Se pudo detectar que la mayoría de las temporeras asocia las dolencias físicas del trabajo de packing a fatiga o sobre-exigencia musculoesqueléticas, resueltas mediante masajes o automedicación. Sin embargo, los efectos de desgaste o daño musculoesquelético son catalogados por las temporeras como efectos de la edad, y no producto del trabajo.

- Variedad de edad y rotación de trabajos: La variedad de rango etario de las trabajadoras temporeras así como de las labores que realizan en la empresa, dificulta la detección de dolencias o daños musculoesqueléticos específicos del trabajo de packing.

- Necesidades económicas: La concepción tradicional presente en la mayoría de las temporeras de que "la mujer aguanta todo" es un factor que incide en la dificultad para la detección de dolencias o malestares. Las temporeras no indican todas las dolencias que pudieran sentir a causa de los trabajos de packing por el miedo a perder su trabajo o porque, simplemente, no lo consideran de relevancia.

- Factores de riesgo psicosocial y salud mental de las temporeras: La alta demanda laboral sumada a exigencias por condición de género (mujer-madre) en el ámbito familiar genera agotamiento psicológico y estrés en trabajadora temporera.

- Diseño y herramientas de los puestos de trabajo: El uso de herramientas y diseños de puestos de trabajo de packing de tipo estándar es un factor de riesgo relacionado al desgaste físico y daño físico de las trabajadoras.

- Implementos de seguridad y protección: La inadecuación de implementos de trabajo genera incomodidad e inseguridad en la trabajadora, lo que incide en: deficiencia en el desempeño de las labores, aparición de hongos en pies, aparición de ampollas, entumecimiento de ciertas zonas del cuerpo, entre otros.
1. Chile. Ministerio de Agricultura. Agricultura Chilena 2014: Una perspectiva a mediano plazo. Santiago: Ministerio de Agricultura; 2005.

2. Gómez M. Temporada de frutas 2005/06 y perspectivas de 2006/2007. Santiago: ODEPA. 2007.

3. Mendoza A, Donoso A. Trabajo agrícola de temporada y su impacto en la Salud. Rev Soc y Equidad. 2011;1-8

4. Lara S. Mercado de trabajo rural, nuevos territorios migratorios y organización de migrantes. Ponencia presentada en el $\mathrm{V}$ Congreso de la Asociación Nacional de Estudios del Trabajo AMET; Oaxtepec, Morelos, México; 2006.

5. Lara S. Nuevas experiencias productivas y nuevas formas de organización flexible del trabajo en la agricultura mexicana. México: Procuraduria AgrariaJuan Pablos Editores; 1998.

6. Caro P. Encrucijadas entre trabajo y familia. Contratos de género y transformaciones sociales en temporeros/as del vino en Casablanca y Mendoza [Tesis de Doctorado]. Santiago de Chile: Universidad de Santiago de Chile; 2012.
7. Riquelme V. Temporeros agricolas: desafíos permanentes. Temas laborales (15). Santiago: Dirección del Trabajo; 2000.

8. Valdés X. La feminización del mercado de trabajo agrícola de Chile Central. Santiago: Centro de Estudios para el Desarrollo de la Mujer; 1988.

9. Vives $A$, Jaramillo $H$. Salud laboral en Chile. Arch prev riesgos labor. 2010;13(3):150-6

10. Orozco R, Chabur S, Montes S, Murillo M, Rubiano D, Sandoval D. Modelo de intervención fisioterapéutica en desórdenes músculo-esqueléticos. Rosario: Facultad de Rehabilitación y Desarrollo Humano-Universidad del Rosario; 2008.

11. Simcox N, Flanagan ME, Camp J, Speilholz P, Snyder K. Musculoskeletal risks in Washington State apple packing companies. Washington: University of Washington; 2001.

12. Fafthallah FA. Musculoskeletal disorders in labor-intensive agriculture. Appl Ergon. 2010;41(6):738-43. 
13. Chile. Ministerio de Salud-Departamento de Salud Ocupacional. Norma técnica de identificación y evaluación de factores de riesgo de Trastornos Musculo Esqueléticos Relacionados al Trabajo (TMERT). Extremidades superiores. Santiago: Ministerio de Salud; 2012. p. 44.

14. Willson A, Caro P. Temporeras de la agroexportación en Chile: tensiones y desafíos asociados a la relación entre la vida laboral y familiar. Mujer y Desarrollo (94). Santiago de Chile: CEPAl; 2010.

15. Caro $P_{1}$ De la Cruz C. Trabajadoras de la agro exportación: costos y consecuencias derivadas de la precariedad del empleo. Frutas y flores de exportación, las condiciones de las trabajadoras en Chile y Colombia. Santiago de Chile: Edics. OXFAM; 2004. p.119-59.

16. Chile. Dirección del Trabajo. Código del Trabajo [en línea]. Santiago: Direccción del Trabajo; 2015 [consultado ago 2015]. Available from: http://www.dt.gob.cl/ legislacion/1611/articles-95516_recurso_1.pdf

17. Chile. Ministerio del Trabajo y Previsión Social. Ley No 16.744. Seguro Social contra Riesgos del Accidentes del Trabajo y Enfermedades Profesionales[en línea]. Santiago: Mlnisterio del Trabajo y previsión Social; 2013 [consultado ago 2015]. Available from: http://www.dt.gob.cl/legislacion/1611/w3- article60854.html

18. Chile. Ministerio del Trabajo y Previsión Social. Decreto Supremo 109. Reglamento de Calificación de accidentes y enfermedades profesionales [en línea]. Santiago: Ministerio del Trabajo y Previsión Social; 2013[consultado ago 2015]. Available from: http://www.dt.gob.cl/legislacion/1611/w3-article $-79400 . h t m l$

19. Chile. Ministerio del Trabajo y Previsión Social. Código Sanitario. Decreto 594, sobre Condiciones sanitarias y ambientales básicas en lugares de trabajo. Santiago: Ministerio del Trabajo y Previsión Social;2013 [consultado ago 2015]. Available from: http://www.dt.gob.cl/legislacion/1611/w3-article-59796.html

20. Chile. Ministerio del Trabajo y Previsión Social. Ley No 20.123 Regula trabajo en régimen de subcontratación, el funcionamiento de las empresas de servicios transitorios y el contrato de trabajo de servicios transitorios. [en línea]. Santiago: Ministerio del Trabajo y Previsión Social; 2013. Available from: http:// www.dt.gob.cl/legislacion/1611/w3-article-94057.html

21. Chile. Ministerio del Trabajo y Previsión Social. Ley No 19.404, Ley de Trabajo Pesado [en línea]. Santiago: Ministerio del Trabajo y Previsión Social; 2013 [consultado ago 2015]. Available from: http://www.dt.gob.cl/legislacion/1611/ w3-article-86873.html

22. Chile. Ministerio del Trabajo y Previsión Social. Ley No 20.005 sobre tipificación y sanción del acoso sexual[en línea]. Santiago: Ministerio del Trabajo y Previsión Social; 2013 [consultado ago 2015]. Available from: http://www.dt.gob.cl/ legislacion/1611/w3-article-80239.html

23. Echeverría M. El reconocimiento de los trastornos de salud mental en el trabajo en Chile. Informe para el Proyecto: Research, policy and practice with regard to work-related mental health problems in Chile: a gender perspective-Proyecto Araucaria. Santiago: CEDEM; 2007.

24. Caro P. Caso de Chile. Capítulo III. In: Baqueros FS, Klein E, editores. Empleo y Condiciones de Trabajo de Mujeres Temporeras Agrícolas. Tomo 1. Roma: CEPAL-OIT-FAO; 2012.

25. AGROCAP. Característica de la oferta de trabajo y de las condiciones de empleo en el sector frutícola de exportación. Santiago de Chile: Pontificia Universidad Católica de Chile-Facultad de Agronomía e Ingenieria Forestal; 2009.

26. Caro P. Contratistas e intermediación laboral en la agricultura de exportación. Santiago: CEDEM; 2005.

27. Brunel S, Moreno N, Blount E. Salud, mujeres y trabajos. Guia para la mejora de las condiciones de salud y trabajo de las mujeres. Madrid: Secretaria Confederal de la mujer-CCOO; 2004.

28. Valdés X. Asuntos privados, problemas públicos. El Estado, la familia y la vida privada en poblaciones rurales en la segunda mitad del siglo XX. Espacios de género: Imaginarios, identidades e historias. Mexicali: UABC; 2006. p.207-38.
29. ANAMURI. Sintesis Primer Congreso Nacional ANAMURI. Con el apoyo de la Agencia de Cooperación Suecia Solidaridad América Latina (SAL). 21-23 mar 2009; Santiago de Chile. Santiago: ANAMURI-SAL; 2009.

30. Caro P. Revalorizando el trabajo de las mujeres en el sector agroexportador: focos y desafíos. Santiago: CEDEM; 2008.

31. Caro P. Diagnóstico de Salud Ocupacional Trabajadoras Temporeras Comuna Coltauco, Región O'Higgins. Santiago: CEDEM; 2009.

32. Valdés $X$ Araujo $K$. Vida privada. Modernización agraria y modernidad. Santiago: CEDEM; 1999.

33. De-Vera Martín V. Gender and global production systems: The casual workers in the Chilean agro-export sector. I Congreso Internacional de Estudios del Desarrollo Desafios de los Estudios del Desarrollo, 14-16 nov 2012; Santander, España. Santander: REEDES; 2013.

34. Díaz X. ¿Malos tiempos para un "buen" trabajo? Calidad del trabajo y género. Santiago: CEDEM; 2009. p.85. (Cuaderno de investigación; 4)

35. Moffat VG. Algunos Factores de Riesgo en la Salud Mental de la Mujer Rural Temporera y su abordaje desde la Terapia Grupal. Rev Psicol. 2004;13(1):103-13.

36. Ruiz Mancilla CF, Méndez Campos MD. Factores de riesgo psicosocial e indicadores de calidad de vida en trabajadoras agricolas de temporada de la séptima región. Talca: Universidad de Talca; 2012. p.97

37. Chile. Ministerio de Salud. Primera Encuesta Nacional de Empleo, Trabajo, Salud y Calidad de Vida de los Trabajadores y Trabajadoras en Chile (ENETS) 2009-2010 [en línea]. Santiago: MINSAL; 2011 [consultado ago 2015]. Available from: http://epi.minsal.cl/estudios-y-encuestas-poblacionales/ encuestas-poblacionales/enets/

38. Chile. Ministerio de Planificación. Encuesta de Caracterización Socioeconómica Nacional (CASEN). Santiago: MIDEPLAN; 2009.

39. Pérez $S$, Jiménez $A$. La falacia del termómetro: Por qué la encuesta CASEN no mide pobreza [en línea]. Santiago: Centro de Investigación Periodistica (CIPER); 2012 [consultado jul 2015]. Available from: http://ciperchile. cl/2012/08/20/la-falacia-del-termometro-por-que-la-encuesta-casen-nomide-pobrezal

40. Engel E. Encuesta CASEN 2011: ¿Qué pasó y qué hacemos? [en línea]. Santiago: CEP; 2012 [consultado jul 2015]. Available from: http://www. cepchile.cl/dms/archivo_5121_3270/03_Presentacion_EEngel.pdf

41. Ruano OM. El trabajo de campo en investigación cualitativa II. Nure Investig. 2007;29(jul-ag):1-4.

42. Ortí Porcar MJ, Zafra Aparici E. La indagación social, herramienta para la mediación social. Castellón: Universitat Jaume I; 2004. p.20.

43. Chile. Ministerio de Salud. Protocolos de vigilancia para trabajadores expuestos a factores de riesgo de trastornos musculoesqueléticos de extremidades superiores relacionadas con el trabajo. Santiago: MINSAL; 2012. p.43

44. Arriagada Donoso KS, Brito García CP. Estado funcional y calidad de vida de pacientes operados artroscópicamente de Manguito Rotador. Santiago: Universidad de Chile; 2010. p.63.

45. Vera-Villarroel P, Silva J, Celis-Atenas K, Pavez P. Evaluación del cuestionario SF-12: verificación de la utilidad de la escala salud mental. Rev méd Chile 2014;142(10):1275-83

46. Vilagut $G$, Valderas JM, Ferrer $M$, Garin 0 , López-Garcia E, Alonso J. Interpretation of SF-36 and SF-12 questionnaires in Spain: physical and mental componentes. Med Clin (Barc). 2008;130(19):726-735.

47. Piqueras OM, Arizaleta LH, Rodríguez JAP. Normas poblacionales de referencia de la versión española del SF-12V2 para la Región de Murcia. Gac Sanit. 2011;25(1):50-61.

48. Kennedy CA, Beaton DE, Solway S, McConnell S, Bombardier C. Disabilities of the Arm, Shoulder and Hand (DASH). The DASH and QuickDASH Outcome Measure User's Manual. 3rd ed. Toronto: Institute for Work \& Health; 2011.

49. USA. Social Security Administration. Mental Health Treatment Study; Final Report 2011. Baltimore: Westat; 2011. p.200. 